\title{
Contingency-based emotional resilience: effort-based reward training and flexible coping lead to adaptive responses to uncertainty in male rats
}

\author{
Kelly G. Lambert*, Molly M. Hyer, Amanda A. Rzucidlo, Timothy Bergeron, Timothy Landis and \\ Massimo Bardi
}

Department of Psychology, Randolph-Macon College, Ashland, VA, USA

Edited by:

Ruud Van Den Bos, Radboud

University Nijmegen, Netherlands

Reviewed by:

Bauke Buwalda, University of

Groningen, Netherlands

Marijn Van Wingerden,

Heinrich-Heine University

Duesseldorf, Germany

Marion Rivalan, Neurocure,

Germany

${ }^{*}$ Correspondence:

Kelly G. Lambert, Department of

Psychology, Copley Science Center

134B, Randolph-Macon College, 304

Caroline Street, Ashland, VA 23005,

USA

e-mail: klambert@rmc.edu
Emotional resilience enhances an animal's ability to maintain physiological allostasis and adaptive responses in the midst of challenges ranging from cognitive uncertainty to chronic stress. In the current study, neurobiological factors related to strategic responses to uncertainty produced by prediction errors were investigated by initially profiling male rats as passive, active or flexible copers ( $n=12$ each group) and assigning to either a contingency-trained or non-contingency trained group. Animals were subsequently trained in a spatial learning task so that problem solving strategies in the final probe task, as well-various biomarkers of brain activation and plasticity in brain areas associated with cognition and emotional regulation, could be assessed. Additionally, fecal samples were collected to further determine markers of stress responsivity and emotional resilience. Results indicated that contingency-trained rats exhibited more adaptive responses in the probe trial (e.g., fewer interrupted grooming sequences and more targeted search strategies) than the noncontingent-trained rats; additionally, increased DHEA/CORT ratios were observed in the contingent-trained animals. Diminished activation of the habenula (i.e., fos-immunoreactivity) was correlated with resilience factors such as increased levels of DHEA metabolites during cognitive training. Of the three coping profiles, flexible copers exhibited enhanced neuroplasticity (i.e., increased dentate gyrus doublecortin-immunoreactivity) compared to the more consistently responding active and passive copers. Thus, in the current study, contingency training via effort-based reward (EBR) training, enhanced by a flexible coping style, provided neurobiological resilience and adaptive responses to prediction errors in the final probe trial. These findings have implications for psychiatric illnesses that are influenced by altered stress responses and decision-making abilities (e.g., depression).

Keywords: resilience, uncertainty, prediction errors, DHEA, contingency training, neuroplasticity markers, coping profiles

\section{INTRODUCTION}

Emotional resilience, an organism's ability to respond to stressful situations in an adaptive manner, provides a neurobiological buffer against the negative effects of stress and leads to decreased susceptibility to psychiatric illnesses (Southwick and Charney, 2012; Wu et al., 2013). The classic learned helplessness research assessing dogs' responses in stressful contexts emphasized the relevance of perceived controllability in emotional resilience. In these early studies, inactivity was viewed as a result of perceived uncontrollability and, subsequently, became a popular model of depression (Overmier and Seligman, 1967; Seligman and Maier, 1967; Abramson et al., 1978). In addition to controllability, cognitive factors such as cognitive reappraisal and cognitive flexibility have emerged more recently as important factors in emotional resilience (Feder et al., 2009; Koolhaas et al., 2011; Colby and Shifren, 2013; McRae et al., 2012).

Extending from the learned helplessness research and focusing on decision-making in the presence of uncertainty, the role of prediction errors has been explored. When an anticipated outcome is not realized (i.e., there is a discrepancy between an expected and observed outcome), also known as a prediction error in these contexts (Bubic et al., 2010; Robinson et al., 2013; Steinberg et al., 2013), it is adaptive for the brain to update relevant contingency probabilities. The uncertainty that accompanies prediction errors serves as a neural prompt for relevant probability upgrades, even when the prediction error occurs in the midst of well-learned response-outcome contingencies (Rushworth and Behrens, 2008). If a response is ultimately perceived as being influential in the production of an anticipated outcome, then an animal has a heightened sense of controllability (Moore et al., 2009). Accordingly, stronger perceived contingencies lead to a heightened capacity for goal-directed behavior (Liljeholm et al., 2011). For example, contingency management is a critical component of Behavioral Activation psychotherapy, an empirically supported therapy for depression (Dimidjian et al., 2011). 
Several brain areas have been implicated in both contingency building and contingency restructuring in the presence of prediction errors. For example, the anterior cingulate cortex (Ragozzino and Rozman, 2007; Rushworth and Behrens, 2008) and the medial prefrontal cortex (Matsumoto and Tanaka, 2004; Behrens et al., 2007; Alexander and Brown, 2011; Egner, 2011) have been associated with the detection of the volatility, or uncertainty, of the parameters associated with a specific environmental context. Further, the insular cortex has been implicated in the neural processing of negative outcomes that promote negative emotional responses so that the aversive response can be avoided in the future (Endepols et al., 2010) as well as the facilitation of adaptive decisions in various challenges (Rebola et al., 2012). Finally, the retrosplenial cortex plays a role in the determination of behavioral shifts for the successful completion of emotional tasks with cognitive demands (Bush et al., 2000; Damasio et al., 2000; Vann et al., 2003, 2009; Vann and Aggleton, 2004; Pothuizen et al., 2008; Bardi et al., 2013) with lesions of this area interfering with the processing of fear-related stimuli such as electric shock (Gabriel and Talk, 2001).

It has also been suggested that the habenula, a structure characterized as an important link connecting the midbrain and forebrain in the regulation of emotional behaviors, influences decision making in uncertain contexts. Lesions of the lateral habenula in animal models result in hyperactive responses in emotional contexts; thus, this brain area may contribute to the suppression of behavioral responsiveness, such as freezing in the presence of prey, in stressful situations (Li et al., 2011). Accordingly, the lateral habenula may contribute to the diminished responsiveness observed in the previously discussed learned helplessness model of depression. In humans, the lateral habenula appears to be hyperactive in depressed patients, as well as in healthy individuals experiencing unwanted negative feedback following a failed performance (Li et al., 2011; Savitz et al., 2011; Henn, 2012).

In recent studies in our laboratory, an alternative to the learned helplessness model, that is, learned persistence, has been investigated as a model of enhanced emotional resilience in the midst of uncertainty related to response outcomes (Lambert, 2006; Bardi et al., 2012, 2013). Specifically, effort-based reward (EBR) training, in which rodents are trained to dig for food rewards in a large arena daily for several weeks, is utilized to build responseoutcome contingencies. When presented with a novel, unsolvable problem-solving task, contingency-trained rats were observed to persist longer on the task than comparable noncontingencytrained animals. Further, the contingency-trained animals had higher dehydroepiandrosterone (DHEA)/corticosteroid (CORT) ratios (Bardi et al., 2012); higher levels of DHEA have been associated with adaptive behavioral responses to stress as well as neurotrophic effects (Karishma and Herbert, 2002). Considering that diminished levels of DHEA have been observed in psychiatric illness such as anxiety, depression and schizophrenia (Morgan et al., 2009), altered DHEA/CORT ratios following EBR training may have contributed to learned persistence in the aforementioned problem solving task.

When contingent-trained rats in our laboratory were exposed to a spatial learning task (the dry land maze, DLM), they also exhibited persistence in the probe trial in which the reward was removed from the previously baited well. In this task, contingency trained rats responded to the prediction error very differently than the rats receiving no contingency training. Specifically, contingency trained rats maintained the strategy of remaining in proximity to the previously baited well, both approaching it faster and spending more time interacting with it, than nontrained animals. Focusing on brain responses, even though the noncontingent-trained animals failed to exhibit an adaptive strategy (i.e., enhanced memory of previously baited well) in the probe trial, they exhibited higher fos activation in the insula, retrosplenial cortex and dentate gyrus of the hippocampus. These results suggested that higher activation in areas involved in emotional and cognitive processing accompanied less efficient responses in this task (Bardi et al., 2013).

In addition to the influence of behavioral training on aspects of decision-making in challenging situations, predisposed individual factors also play an influential role. Although the stress response was once thought to be generalized and non-specific (Selye, 1936), it has become increasingly clear that individual differences in stress responsiveness exist. For example, varying responses to stressors, or coping styles, have been identified among members of the same species (Koolhaas et al., 2007). Coping strategies are considered effective when they provide neurobiological adaptations that mitigate threat and enhance survival in threatening situations (Wechsler, 1995). When approach responses to novel objects are assessed in young rats, some animals readily approach the objects whereas some avoid the novel objects. Interestingly, uninhibited rats experience a faster recovery of corticosteroids following a stressful encounter, suggesting that they are less likely to experience allostatic load and an unhealthy stress response. Because the responses of these animals remained consistent when assessed at different developmental stages, these coping response strategies are viewed as an emotional trait that is persistent across the animal's lifetime (Cavigelli and McClintock, 2003; Cavigelli et al., 2007).

Building upon research determining passive and active coping styles in piglets (Koolhaas et al., 1999), a back-test restraint assessment was adapted for rodents to determine predispositions for both consistent and variable, or flexible, coping strategies (Lambert, 2006; Bardi et al., 2012, 2013). In one study, rats were profiled as active, passive and flexible copers and exposed to 3 weeks of chronic unpredicatable stress. Focusing on apparent neurobiological adaptations, the flexible coping rats had higher levels of Neuropeptide Y (known for its role in emotional resilience) in the basolateral amygdala and bed nucleus of the stria terminalis (Hawley et al., 2010; McGuire et al., 2011). Subsequent research in our laboratory has also confirmed the role of hippocampal NPY-immunoreactive cells in various coping profiles with flexible copers exhibiting higher levels in the CA1 subfield than their passive and active counterparts (Bardi et al., 2012).

Taking these findings into consideration, the purpose of the current study was to investigate behavioral and neurobiological responses in rodents exposed to uncertainty in a decision-making task. Of interest was the animal's response to uncertainty related to a prediction error in the cognitive task and the degree of response flexibility, a response that has been interpreted as adaptive in such situations (Donegan et al., 2013; Klanker et al., 2013; 
Rivalan et al., 2013). Specifically, male Long-Evans rats were profiled as active, passive or flexible copers then exposed to either 4 weeks of EBR contingency training or noncontingency training. Following contingency training, both contingent and noncontingent groups were trained in the Dry Land Maze so that, following learning acquisition, decision-making strategies could be observed in response to a prediction error (i.e., negative surprise) in the probe trial. In addition to relevant behavioral responses, the animals' brains were assessed for various forms of activation throughout several brain areas known to facilitate strategic decision-making in emotional contexts. Further, with the established role of neuroplasticity in both cognitive responses and emotional resilience (e.g., protection against depression symptoms), neuroplasticity in various hippocampal areas was also assessed in the current study (Krishnan and Nestler, 2013). It was hypothesized that predisposed flexible coping strategies, as well as contingency training, would result in more adaptive decisionmaking strategies (in this case, the presence of varied and flexible responses) and neurobiological responses consistent with enhanced survival (e.g., avoiding threats and excessive allostatic loads).

\section{METHODS AND MATERIALS ANIMALS}

Fifty male Long-Evans rats were ordered from Harlan Tekland (Madison WI USA) and arrived at 21-23 days of age. At the time of arrival they were housed five animals per cage $(48 \times 26 \times$ $21 \mathrm{~cm}$ ) with corncob bedding and food and water provided ad libitum. A $12 \mathrm{~h}: 12 \mathrm{~h}$ light dark schedule was maintained with lights on at 6:00 am. Following 17 days of habituation to the laboratory, animals were matched with a novel cage-mate with the same coping profile (active, passive or flexible; see below for coping profile assessment). At that time one of the two animals was assigned to the EBR (EBR) contingency-training group (C-T) and one to the control non-contingent training group (NC-T). See Figure 1 for timeline of behavioral procedures described below.

\section{COPING PROFILE ASSESSMENT}

Two days following arrival to the laboratory, coping profile assessments were conducted in the colony room between the times of 1:30 and 3:30 pm with each session videotaped for subsequent confirmation of behavior. During the assessment, each animal was gently restrained on its back for $1 \mathrm{~min}$ so that the number of escape attempts (or wiggles) could be quantified (see Hawley et al., 2010). Seven days later the same assessment was conducted; however, the animals were tested in a different order than the first assessment to avoid any confounding sequencing effects. Once the two sessions were observed and scored, the total number of escape attempts was used as the criterion score for the determination of coping profile. Considering that the greatest number of escape attempts for each session was 11 responses, animals with fewer than 6 responses were categorized in the passive coping group and those with 6 or more responses were categorized as active copers. If the response number stayed in the same category in the second assessment 1 week later then its final placement was in that respective category. However, if the animal switched from one category to another (in either direction), it was classified as a flexible coper. The most representative animals were selected from the 50 animals so that each group consisted of 5-6 animals with the lowest and highest escape attempts (passive copers and active copers, respectively); accordingly, the flexible copers with the largest differences in escape attempts were assigned to the flexible coping groups. In total, 34 animals placed in six groups (two training groups $\times 3$ coping groups) were used in the current study. Animals were maintained in accordance with the Randolph-Macon College Institutional Animal Care and Use Committee.

\section{EFFORT BASED REWARD (EBR) TRAINING}

Half of each coping group was assigned to the contingent-trained (C-T) group and half to the noncontingent-trained (NC-T) group. Further, half of the animals in each group commenced training 1 week later than the other animals to evenly space out training, testing, and histological processing for the investigators. Twenty days after arrival to the lab, the first group of animals commenced training. Food was removed from the cages at 9:00 am on the first day of training and each rat received a piece of sweet cereal (i.e., froot loop ${ }^{\circledR}$ cereal) to habituate to the taste of the food reward used in the task (see Bardi et al., 2012, 2013). Approximately $4 \mathrm{~h}$ later, training began by placing the rats individually into an open arena $(122 \times 91 \times 51 \mathrm{~cm})$ built of wood and covered with gray linoleum. Corn-cob bedding was placed on the floor of the arena at a depth of approximately $7 \mathrm{~cm}$. For the $\mathrm{C}$ - $\mathrm{T}$ group, the bedding was arranged into four mounds that were approximately $10 \mathrm{~cm}$ tall with the locations of the mounds varied each day. In the early trials, a half piece of cereal was placed on the top of the mound so that it could be easily detected by the rats. The $\mathrm{C}-\mathrm{T}$ animals were subsequently placed in a consistent corner of the arena and were given $6 \mathrm{~min}$ to explore the arena and mounds with food rewards; however, if all the froot loops were retrieved prior to the 6 min period, the animal was removed from the arena so it wouldn't experience the mounds with no rewards and compromise the contingency associations between digging and rewards. Following training, each animal was weighed and placed in its home cage. Between sessions, the bedding was redistributed to mask the previous animals' scent and paths for the

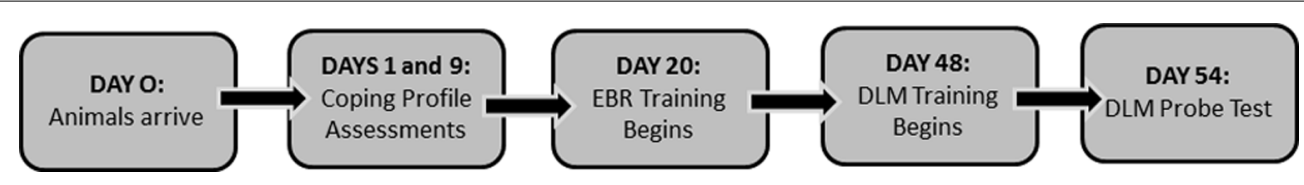

FIGURE 1 | Timeline for behavioral procedures utilized in the current study. Animals arrived in the laboratory at approximately 23 days of age then were exposed to the sequence of events in this timeline. 
subsequent N-CT animal. Each N-CT animal was time-yoked to the $\mathrm{C}$ - $\mathrm{T}$ animal and placed in the arena with mounds but the cereal rewards were placed collectively in the open so that the $\mathrm{N}$-CT could access the rewards with minimal effort. After the first week, the N-CT animals were also yoked to the C-T animals on the number of rewards consumed so that, after week one, the $\mathrm{C}-\mathrm{T}$ and NC-T animals were both time- and reward-yoked. Also beginning during the second week of training, the cereal pieces were slightly hidden in the top of each mound, then at the beginning of the third week the rewards here hidden $2.5 \mathrm{~cm}$ in the mounds and, by the fourth week, they were positioned near the bottom of the mounds. During training, since the food was removed for approximately $4 \mathrm{~h}$ each day (with food available ad libitum for the rest of the day), the animals were weighed every third day and monitored for appropriate growth to assure that they were maintaining healthy weights with no animal exceeding more than a $10 \%$ weight loss. This training continued for 4 weeks (with no training on weekend days).

\section{DRY LAND MAZE (DLM) TRAINING AND PROBLEM SOLVING ASSESSMENT}

On the day following the completion of EBR contingency training, animals commenced habituation training for the DLM task (see Franssen et al., 2011; Bardi et al., 2012). During this training the same food restriction protocol as the EBR training was used and time of testing was shifted $2 \mathrm{~h}$ later. A circular apparatus measuring $124.5 \mathrm{~cm}$ in diameter and $40.5 \mathrm{~cm}$ in height was used for training and testing. Eight plastic wells $(2 \mathrm{~cm}$ in diameter and $1 \mathrm{~cm}$ in height) were secured on the bottom of the arena positioned equidistantly along the periphery. During habituation day 1, all 8 wells were baited with one-quarter of a cereal piece. Animals were once again given $6 \mathrm{~min}$ to locate and consume the food rewards. On days 2 and 3 of habituation training, four wells (every other one) and two wells (two of the four) were baited, respectively, for the $6 \mathrm{~min}$ trials. On the following day, animals were exposed to the Acquisition Trial which consisted of only one of the two previously baited wells being baited for the testing and probe trial assessment. For the three subsequent test days, the same single well was baited and each animal was exposed to three 3-min trials with an inter-trial interval of $1 \mathrm{~min}$. Animals were placed in varying start positions throughout the test trials; however, the start positions were consistent for all animals. On the day following the second day of testing, animals were exposed to the probe trial to assess reactions to prediction errors by being placed in the DLM arena for $5 \mathrm{~min}$ with no reward in the previously baited well. Each animal's behavior was videotaped for subsequent behavioral analysis of the following behaviors: latency to approach the previously baited well; time spent in proximity to the baited well; rearing; investigation of other wells and freezing responses (see Table 1 for ethogram of behaviors scored in the probe trial). Together with these traditional behavioral measures, we also recorded behavioral sequences and transitions for both movements and grooming. Distractions or interruptions of sequenced behavioral responses have been associated with heightened anxiety (Bardi et al., 2011). The common and widespread use of averaged measures taken at specific time intervals is useful for collecting snap-shots summaries of behavior, but
Table 1 | Ethogram of the behavioral measures.

\begin{tabular}{|c|c|}
\hline $\begin{array}{l}\text { Behavioral } \\
\text { measure }\end{array}$ & Definition \\
\hline Freezing & Suddenly becoming rigid or motionless. Fear reaction \\
\hline Exploration & $\begin{array}{l}\text { Information gathering necessary for the formation of } \\
\text { spatial representation (touching or sniffing the } \\
\text { environment) }\end{array}$ \\
\hline Scratching & $\begin{array}{l}\text { Rapid movements of hand or foot to rake or pick the } \\
\text { skin of the body }\end{array}$ \\
\hline Grooming & $\begin{array}{l}\text { Complex strings of movements to clean and maintain } \\
\text { the fur and skin of the body (wiping, licking, and } \\
\text { strokes). Sequential chain }\end{array}$ \\
\hline $\begin{array}{l}\text { Grooming } \\
\text { interrupted }\end{array}$ & $\begin{array}{l}\text { Non-chain sequences of grooming (incomplete } \\
\text { sequences or random sequences) }\end{array}$ \\
\hline Rearing & Standing up on the hind legs sniffing \\
\hline Proximity & Closer to one body length to the previously baited well \\
\hline Jumping & Sudden movements spring of the ground \\
\hline Inversion & Any change in the direction of movement \\
\hline Stereotype & Any form of rapidly repeated movement \\
\hline Transition & Any change in behavior (i.e., from freezing to exploring) \\
\hline
\end{tabular}

they often fail to disentangle more subtle variations in behavior (Bardi et al., 2011). Therefore, we calculated the number of interrupted grooming sequences (either an incomplete chain or a chain diverging from the typical cephalocaudal structure), the number of inversions (i.e., changes in directions while in movement such as walking from clockwise to anticlockwise), the number of stereotypical movements, and the number of behavioral transitions (e.g., from running to exploring).

\section{PHYSIOLOGICAL RESPONSES}

To assess the physiological responses to the behavioral tests, CORT and DHEA metabolized in excreta were assessed using fresh fecal samples. Samples were collected at approximately 9:00 am with the baseline sample collected during the second week of EBR training (following a weekend nontraining day with no food restriction) and, for the test sample, $12 \mathrm{~h}$ following the initial acquisition trial of the DLM, representing a challenging phase of the DLM training (see Bardi et al., 2011 for parametric validation of times used in fecal sample collection). Thus, the test samples were collected following a rather mild cognitive challenge phase of the DLM task and were not perceived as fully engaged stress responses. Animals were briefly isolated (no more than $10 \mathrm{~min}$ for each animal was necessary to collect samples) and samples $(0.1 \mathrm{~g}$ each) were collected from each animal and frozen unmixed in sealed containers at $-70^{\circ} \mathrm{C}$ until assaying. A total of 48 samples were collected and saved for CORT and DHEA extraction and assay procedures.

Prior to extraction, previously collected fecal samples were thawed at room temperature and placed in a glass tube with $1 \mathrm{ml}$ of $100 \%$ methanol. The contents of the tube were then mixed via vortex (Vortex Genie 2, Scientific Industries, Inc.) for approximately $30 \mathrm{~s}$. Next, the tube was centrifuged for $10 \mathrm{~min}$ at $2500 \mathrm{rpm}$. Using a transfer pipette, the sample was transferred to a $13 \times 100 \mathrm{~mm}$ glass test tube. The final step of extraction 
procedures was to dilute the sample in $\mathrm{MeOH}$ (concentration 1:20) in an EIA buffer. Assay procedures were carried out using materials and protocols provided by an Enzyme ImmunoAssay (EIA) kit (Assay Designs, Anne Arbor, Michigan). Correlate-EIA sample readings were completed using an automated microplate reader (BioTek, Winooski, VT, model \# EL $\times$ 800) and the KC Junior software (BioTek, Winooski, VT, version 1.3, Part $5270501)$. Readings were assessed at a wavelength of $405 \lambda$. The cross-reactivity of the cortisol kit, as reported by the manufacturer, was $100 \%$ with cortisol and prednisolone (122\%), 27.68\% with corticosterone, $4 \%$ with 11-deoxycortisol, and negligible for other steroids (under 1\%). The cross-reactivity of the DHEA kit was 100\% with DHEA, 30\% with DHEA sulfate, and considered negligible for other steroids (under 1\% of androstenedione, androsterone, and so forth). Sensitivity of the kit was $56.80 \mathrm{pg} / \mathrm{mL}$ for cortisol and $2.90 \mathrm{pg} / \mathrm{mL}$ for DHEA.

To demonstrate parallelism and accuracy of the CORT and DHEA assays, each of five randomly selected fecal samples were serially diluted (1:2-1:16). Percentage-binding data from the standard curve were plotted against logarithmic transformations of their dosages and the resulting regression equation was compared to those of the dilution sequences. To assess dose response, another five samples were randomly selected and unlabeled CORT or DHEA was added to $10 \mathrm{~L}$ sample aliquots in increasing doses: $0,2.5,10,40$, and $160 \mathrm{pg} /$ tube.

The dose response study generated a curve with a slope of $1.03\left(r^{2}=0.975\right)$ for CORT and a curve with slope of $1.01\left(r^{2}=\right.$ 0.997 ) for DHEA. Mean recoveries were $95.21 \%$ for CORT and $97.19 \%$ for DHEA over a range of 2.5 to $160 \mathrm{pg}$. The slopes generated from the serially diluted samples in the parallelism study were not different from the standard curve slope (CORT: $p=$ 0.866 , DHEA: $p=0.815)$. Intra- and interassay coefficients of variations were 4.43 and $11.15 \%$ for CORT and 5.33 and $9.87 \%$ for DHEA.

\section{HISTOLOGICAL PREPARATION}

Ninety minutes following the 5-min probe trial, animals were anesthetized and perfused to detect fos-immunoreactivity as well as other relevant neural markers. Specifically, individual animals were exposed to $1 \mathrm{~mL}$ of Halothane liquid (Sigma-Aldrich; St. Louis MO) until respiratory rate slowed and were subsequently given an intraperitoneal injection of $0.2 \mathrm{~mL}$ sodium pentabarbitol at an overdose of $50 \mathrm{mg} / \mathrm{Kg}$ and transcardially perfused at $40 \mathrm{~mL} / \mathrm{min}$ using a MasterFlex L/S perfusion pump initially with $100 \mathrm{~mL}$ phosphate-buffered saline solution, then with $200 \mathrm{~mL} 4 \%$ paraformaldehyde. Following extraction, brains were post-fixed in $4 \%$ paraformaldehyde overnight at $4^{\circ} \mathrm{C}$, then transferred to a $10 \%$ sucrose solution for $24 \mathrm{~h}$ at $4^{\circ} \mathrm{C}$ followed by a $20 \%$ sucrose solution at $4^{\circ} \mathrm{C}$ until the time of sectioning. Brains were subsequently sectioned at $-25^{\circ} \mathrm{C}$ using a HM525 Microm cryostat at the appropriate Bregma position for each targeted brain (using Paxinos and Watson, 2007). For all sections, every 7th section was used to avoid double-counting cells in serial sections and to provide enough tissue for all the histological protocols.

Six free-floating sections $(40 \mu \mathrm{m}$ thickness) were collected through the following brain areas: Lateral habenlua; hippocampus (dentate gyrus, CA1, CA2, and CA3); and cortex [insular, restrosplenial, and piriform areas (the piriform cortex was assessed due to its projections to both the limbic and cortical structures; Johnson et al., 2000)]. See Figure 2 for brain areas and representative photomicrographs of habenula and hippocampal neural markers. For fos-immunreactivity assessment in the habenula, insula and piriform cortical areas, following a $10 \mathrm{~min}$ wash in $0.1 \%$ hydrogen peroxide to quench endogenous peroxidase activity, sections were blocked for $30 \mathrm{~min}$ in $10 \%$ normal goat serum (Vector, Burlingame, CA) in PBST (0.3\% Triton-X, Spectrum Chemical: Cardena, CA). Sections were subsequently incubated in c-fos primary antibody (1:10,000; Immunostar, Inc., Hudson, WI, USA) overnight at $4^{\circ} \mathrm{C}$ followed by $1 \mathrm{~h}$ exposure to goat anti-rabbit secondary antibody (Vector) at 1:1500, and further processed with a standard Vectastain ABC kit (modified with Bovine Triton-X PBS; Vector). A similar protocol was used for BDNF (Abcam, Inc., Cambridge, MA USA; primary $1: 1000$ and secondary 1:250) and nestin (primary antibody dilution of 1:400 and secondary dilution of 1:200; Developmental Studies Hybridoma Bank, University of Iowa, Iowa City, IA USA) to assess neurotrophic activity and neuronal restructuring (Hendrickson et al., 2011), respectively. All sections were visualized with DAB peroxidase substrate and then cleared through a series of 70, 95, and 100\% EtOh and Citrisolv (Fisher Scientific, Fair Lawn, NJ, USA) washes and coverslipped using Permount (Fisher Scientific).

For fluorescent immunocytochemistry, sections were incubated in $0.3 \%$ trisodium citrate solution for $10 \mathrm{~min}$ in a $90^{\circ} \mathrm{C}$ water bath. Following three 5-min PBS-Tween washes, sections were exposed to $6 \%$ normal donkey serum in PBS-Tween for 30 min (Spectrum Chemical, New Brunswick, NJ, USA), and then incubated overnight at $4^{\circ} \mathrm{C}$ in a 1:1000 dilution of rabbit anti-DCX (Abcam, Inc., Cambridge, MA, USA). Following three PBS-Tween 20 min washes, sections were exposed to the secondary anti-rabbit antibody at a dilution of 1:200 (Abcam). GFAP sections were treated in a similar manner but exposed to primary anti-rabbit antibody at 1:1000 dilution and secondary rhodamine red anti-rabbit antibody at 1:200 (Abcam, Inc.). GFAP slides were counterstained with DAPI (0.01\%; Abcam) for $1 \mathrm{~h}$ in the dark.

\section{NEURAL QUANTIFICATION}

Prior to being quantified, all slides were recoded so that experimenters would be blind to experimental conditions. A Zeiss Axioskop light microscope (Carl Zeiss, Oberkochen, Germany) and Neurolucida software (Microbrightfield, Inc., Williston, VT, USA) were used to quantify fos-immunoreactive cells in the lateral habenula (at $10 \times$ magnification with a $300 \times 300 \mu \mathrm{m}$ grid), the piriform cortex (at $10 \times$ magnification with a $500 \times 300 \mu \mathrm{m}$ grid), and in the insula $(500 \times 500 \mu \mathrm{m}$ at $10 \times)$. In these areas, cells were marked with a computer-generated colored symbol and quantified for each section.

For the remaining markers, a BA400 light microscope (Motic, Richmond, BC, Canada) was used for the standard neuroquantification of BDNF (using a $125 \mu \mathrm{m}$ radius circle at $20 \times$ magnification) and CA1, CA2, and CA3 nestin (using a $135 \times 135 \mu \mathrm{m}$ area at $40 \times$ magnification) as well as for fluorescent neuroquantification for doublecortin in the dentate gyrus $(270 \times 270 \mu \mathrm{m}$ at $10 \times$ magnification) and GFAP and DAPI in the retrosplenial cortex 


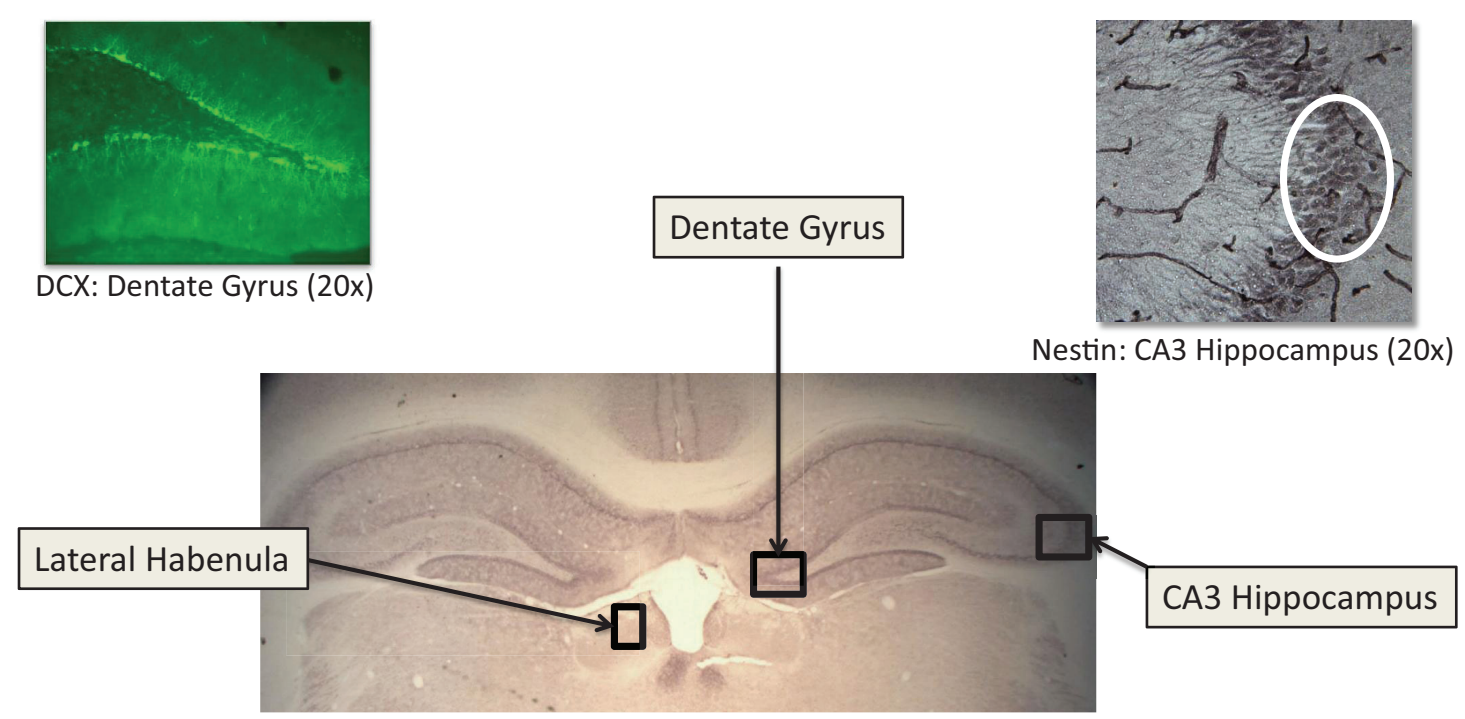

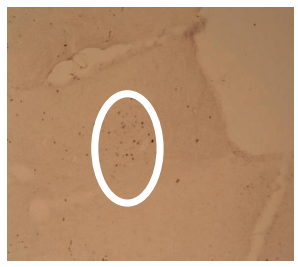

Fos: Lateral Habenula (20x)

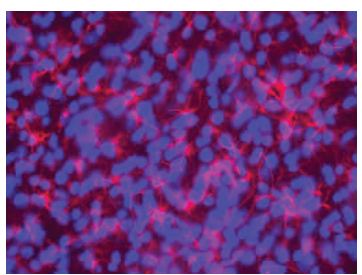

DAPI/GFAP: Lateral Habenula (40x)

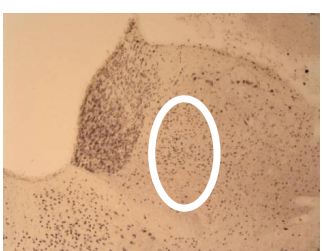

BDNF: Lateral Habenula (10x)

FIGURE 2 | Representative photomicrographs of relevant immunoreactive tissue in the lateral habenula and hippocampus (dentate gyrus and CA3 area. See Results for statistical findings for each area.

$(135 \times 135 \mu \mathrm{m}$ at $40 \times$ magnification). In all cases, quantification was accomplished using light-thresholding software (Bioquant Life Sciences, Nashville, TN, USA) so that the proportion of positively stained tissue to nonstained tissue could be determined for each visual field. For the determination of nestin-immunoreactive cells, the nestin-immunoreactive blood vessels were subtracted from the visual field so that only pyramidal neuronal cells were quantified.

\section{STATISTICAL ANALYSIS}

For all dependent variables, a 2 × 3 General Linear Model (GLM) analysis was used to determine the effects of EBR training (two levels: contingent training present or not) and coping profile (3 levels: active, passive and flexible) respectively. For each analysis, the $\alpha$-value was set at 0.05. Following the GLM analyses, appropriate Tukey post-hoc tests and Pearson's correlations were conducted to further elucidate relationships among dependent variables.

A multi-dimensional scaling (MDS) analysis was conducted in order to provide a model of independent associations among the variables. We preferred MDS to other multivariate techniques, such as MANOVA or factor analysis, since MDS does not require the data to be multi-normally distributed, a condition rarely satisfied with behavioral measures. MDS is a data reduction technique used to uncover a "hidden structure" to a set of data (Kruskal and Wish, 1978). MDS refers to graphical models that provide a spatial representation of the similarity structure of variables. Using correlations, the relationships (i.e., proximities) among variables can be displayed graphically. Because the variables are represented by a set of points in a two or higher dimensional space (a map), the closer two or more variables are on the map, the more highly correlated they are, while the farther apart they are, the less correlated they are. In order to map all of the variables into a desired space (two dimensional or greater), a certain lack of fit is accepted. This lack of fit is referred to as the s-stress. The values of sstress range from 0 (perfect fit) to 1 (worst possible fit). Thus, the aim of MDS is to find a map of the variables that minimizes the s-stress for a given number of dimensions. The number of dimensions can be linked to the number of latent underlying factors in the dataset. Thus, when choosing the number of dimensions to represent the data, one must consider (1) the number of variables in the model, (2) the lack of fit (s-stress value), given the number of dimensions, (3) an index of fit of the model $\left(r^{2}-\right.$ value), and (4) interpretability of the dimensions (Manly, 1994). The first point addresses the fact that for each dimension of the data, there should be approximately 4 variables entered into the model. Thus, for a 2-dimensional map, approximately 8 variables should be used. The second point addresses how well the 
MDS map actually "fits" the data. Stress values below 0.15 are typically deemed acceptable. The third point addresses the variance accounted for within the model. As is the case with any regression analysis, the amount of variance being accounted for is an important consideration. Typically, $r^{2}$-values of 0.8 or higher are desirable. Finally, one must select a solution based on interpretability of the dimensions. Parsimony is crucial to interpreting the "map" of any given dataset. In sum, MDS is a technique that provides additional information about the "structure" of a dataset, which is not possible with standard parametric statistical techniques.

\section{RESULTS \\ BEHAVIORAL RESULTS}

For the variable of frequency of exploration bouts during the DLM probe test, the GLM revealed a significant interaction between EBR training and coping profile $\left[F_{(2,28)}=3.38 ; p=\right.$ 0.048]. Specifically, as seen if Figure 3A, flexible C-T rats exhibited fewer exploration bouts than flexible NC-T rats (Tukey post-hoc test: $p=0.03$ ). Additionally, a significant main effect for EBR indicated a lower overall frequency of exploratory bouts for the contingent trained animals $\left[F_{(1,28)}=4.64 ; p=0.04\right]$. No differences were observed in the overall duration of exploration [corrected model: $F_{(5,28)}=0.24, \mathrm{~ns}$ ].

Focusing on the duration of time spent in proximity to the previously baited well in the DLM Probe test, a main effect for EBR training was observed $\left[F_{(1,28)}=7.69 ; p=0.01\right]$. Specifically, C$\mathrm{T}$ animals spent $140 \%$ more time than $\mathrm{NC}-\mathrm{T}$ rats in proximity to the targeted well (see Figure 3B). C-T animals also visited the previously baited well significantly more often than the NC-T animals $\left[F_{(1,28)}=7.297 p=0.012\right.$; see Figure 3C]. Considering all of the eight wells in the DLM arena, the NC-T rats visited a higher number of wells in the arena than the C-T animals $\left[F_{(1,28)}=\right.$ $4.29 ; p=0.048$; NC-T animals visited 11 wells compared to 7.6 for C-T animals; see Figure 3D].

Although no differences were found in the total duration or frequency of self-grooming a significant effect of EBR training was found in the frequency of interruptions in the grooming sequence $\left[F_{(1,28)}=11.18 ; p=0.002\right]$. As seen in Figure 3E, the $\mathrm{N}-\mathrm{CT}$ animals were more than twice as likely to exhibit an interrupted grooming sequence. EBR training was also found to have an effect on rearing responses in strategic areas of the DLM arena, namely in either the center or in proximity to the wells $\left[F_{(1,28)}=4.65 ; p=0.04\right.$; see Figure 3F $]$. In this case, the C-T rats exhibited approximately three times more rearing responses than their NC-T counterparts. Neither EBR training nor coping profile affected the remaining variables that were scored, including freezing, jumping, scratches, and stretch-attend responses (all $p$-values $>0.18$ ).

\section{ENDOCRINE RESULTS}

CORT metabolites decreased significantly between the baseline levels collected at the beginning phases of EBR training and after EBR training following the acquisition trial of DLM testing, representing a phase of more challenging cognitive training $\left[F_{(1,28)}=7.08, p=0.013\right]$. In contrast, DHEA metabolites significantly increased between the baseline levels to the DLM trial $\left[F_{(2,18)}=6.60, p=0.023\right]$. After the DLM acquisition trial, DHEA/CORT ratios were calculated and C-T animals had a significantly higher ratio than the NC-T rats $\left[F_{(1,28)}=5.57\right.$; $p=0.026]$. Although no significant interaction was observed, planned comparisons revealed a significant effect of training in the flexible training group with $\mathrm{C}-\mathrm{T}$ animals having a higher ratio than NC-T animals $\left[t_{(8)}=2.56 ; p=0.03\right]$. See Figure 4 for the DHEA/CORT metabolite levels in the test sample following the acquisition trial in the DLM.

\section{NEURAL RESULTS}

A significant coping effect was observed in the percentage of doublecortin-immunoreactive tissue in the dentate gyrus visual field $\left[F_{(2,28)}=7.55 ; p=0.002\right]$. As seen in Figure $5 \mathrm{~A}$, the flexible coping animals had about $66 \%$ more DCX-immunoreactive tissue than the passive and active groups. Focusing on another marker of neuroplasticity, a significant interaction was observed in CA3 nestin $\left[F_{(2,28)}=3.82 ; p=0.034\right]$. In this case, whereas contingency training didn't impact the active and passive coping groups, it reduced nestin-immunoreactivity in the flexible animals (see Figure 5B). Although not reaching statistical significance $\left[F_{(2,28)}=2.49 ; p=0.106\right]$, flexible animals had higher neuron/glia ratios in the lateral habenula (13\% higher than active copers and $21 \%$ higher than passive copers).

Whereas no significant effects of EBR training or coping profiles were observed in the fos-immunoreactive data taken from the habenula, insula and piriform cortex, a few interesting significant correlations between fos-immunoreactivity in these brain areas and the neuroplasticity measures (i.e., nestin in CA2, BDNF in habenula and DCX in the dentate gyrus), as well as with the DHEA/CORT ratio data, were observed (see Table 2). The endocrine data in Tables 2 and 3 represent levels following the cognitively challenging acquisition trial in the DLM training. The DHEA/CORT ratio was positively correlated with DCXimmunoreactive cells in the dentate gyrus $(p=0.04)$; further, a nonsignificant trend was observed suggesting an inverse correlation between DHEA/CORT ratios and fos-immunoreactive cells in the habenula $(p=0.09)$. Focusing on the CORT levels, a significant positive correlation was observed with the nestin CA2 fosimmunoreactive cells ( $p=0.05$ ); further, a nonsignificant inverse relationship was observed between immunoreactive-BDNF tissue in the lateral habenula and CORT $(p=0.06)$. Finally, a significant negative correlation was observed between DHEA levels and fos-immunoreactive cells in the lateral habenula $(p=0.03)$.

\section{INTEGRATIVE MULTIDIMENSIONAL SCALING MAP}

To take into account the multivariate association among measures assessing different systems (neural, endocrine, and behavioral measures) a multidimentional scaling (MDS) analysis was used. The MDS technique generated Figure 6. The Kruskal's stress index determined a stress value equal to 0.101 , indicating a good fit between the dimensions and the mapped distances. The $R^{2}$-value designated that $95.6 \%$ of the variance in the data was explained by the model. Based on the two dimensions, the responses to the EBR conditions were divided into two major clusters, indicated in the figure by a line. The lower quadrants represent noncontingent animals that displayed higher BDNF 


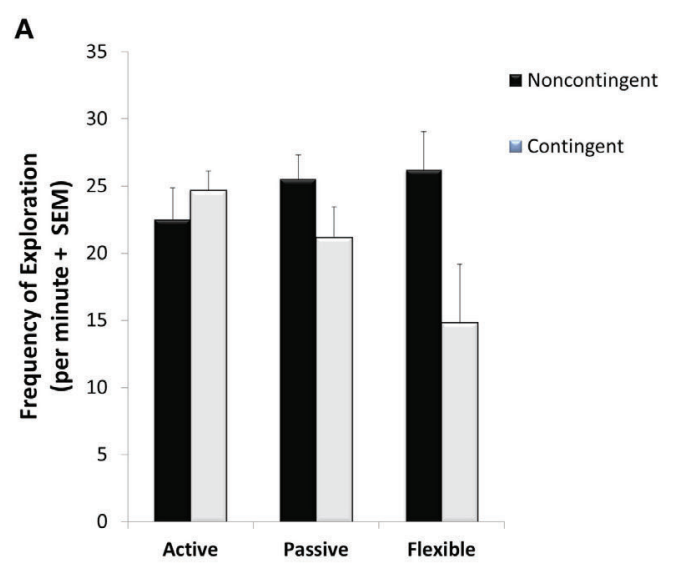

B

C

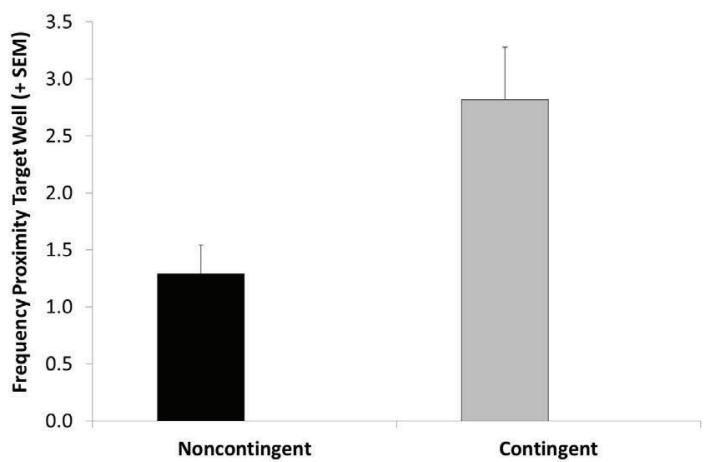

E

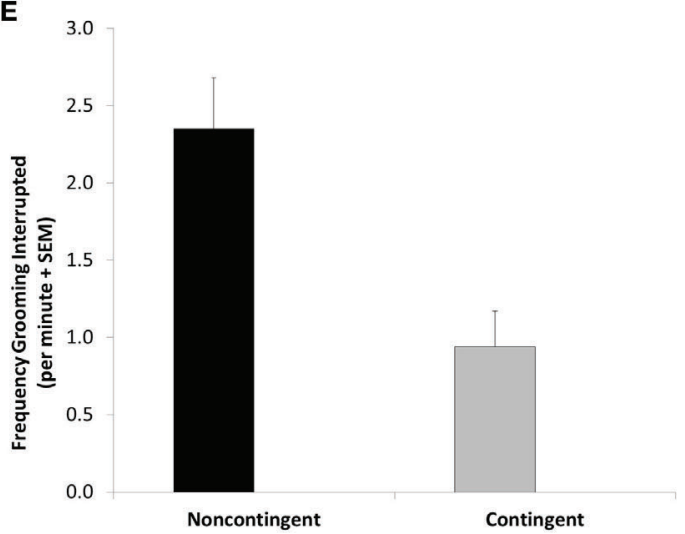

FIGURE 3 | Probe trial behavior. During the 5-min probe trial flexible contingent animals engaged in less exploratory behavior (A) further contingent animals spent more time in proximity to the previously baited well (B) visited the previously baited well more frequently (C)

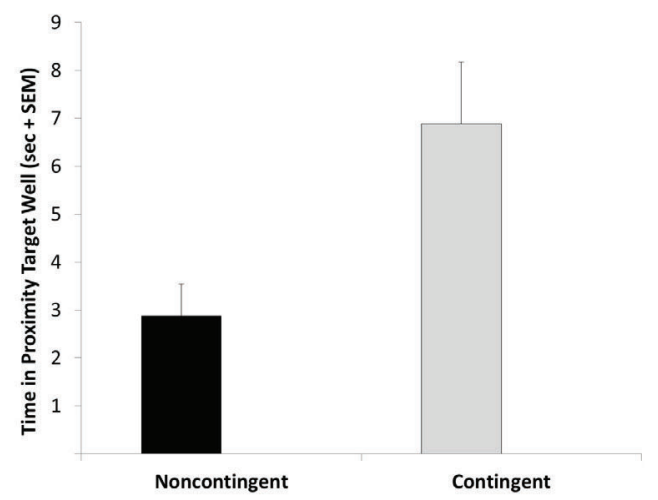

D

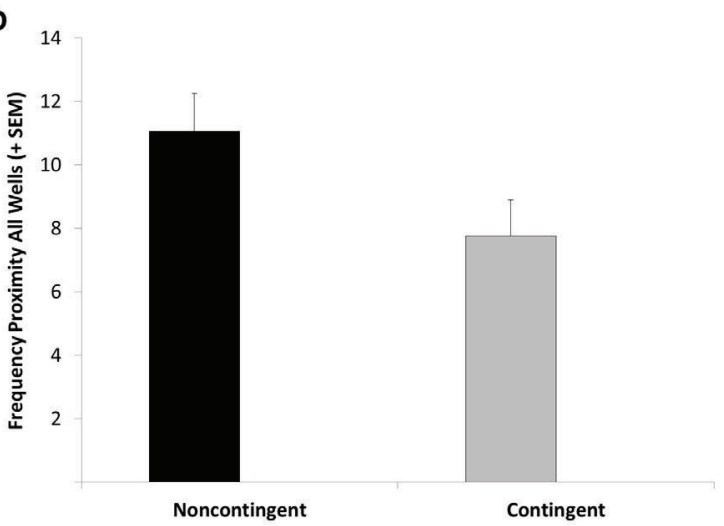

$\mathbf{F}$

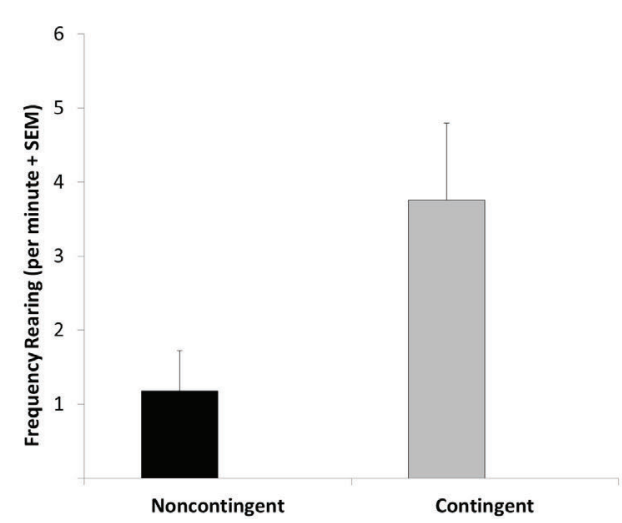

visited the non-baited wells less frequently (D) exhibited fewer interrupted grooming sequences (E) and more rearing responses (F) than their noncontingent counterparts. See Results for specific statistical outcomes. expression in the habenula (BDNF_haben), together with higher level of grooming interrupted (Gr_Int), and explorative behavior (Expl). The second group, in the upper quadrants, was characterized by contingent animals displaying higher neuron to glial cells ratio in the habenula (Ratio_Habe), lower nestin activation in the CA3 (Nestin_CA3), a higher DHEA/CORT ratio (DC_ratio), more time spent in proximity of the previously baited well (Prox_5), and rearing (Rearing).

\section{DISCUSSION}

In the current study, both predisposed coping strategies and contingency training were utilized to determine their impact on the animals' neurobiological and behavioral responses to uncertainty related to prediction errors. The results indicated that EBR contingency training modified targeted neurobiological factors associated with enhanced emotional resilience. Further, the identification of predisposed coping strategies highlighted 


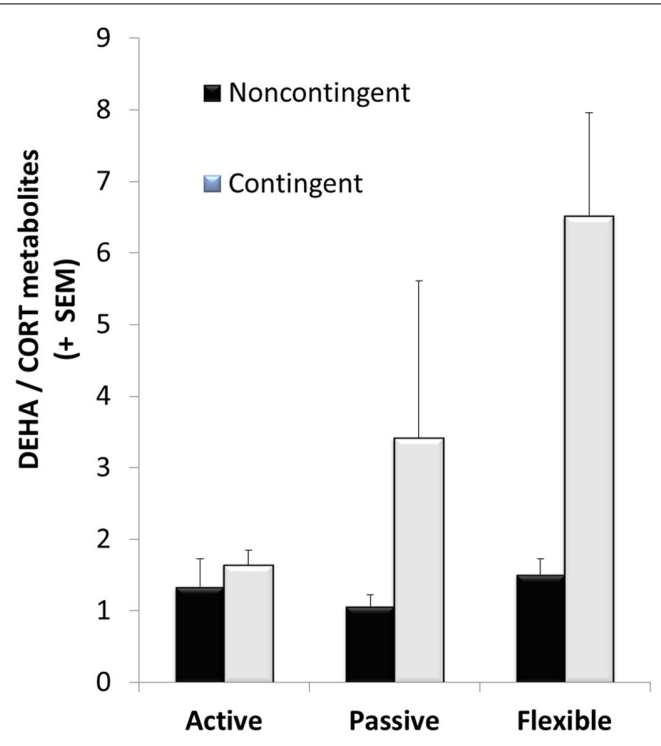

FIGURE 4 | DHEA/CORT metabolite ratios were differentially affected after the acquisition trial in the dry land maze; although there was high variability in the varying coping groups, only a significant main effect for contingency training was observed (with mean values of $3.69 \pm 1.11$ and $\mathbf{1 . 2 9} \pm \mathbf{0 . 1 6}$ for the contingent and noncontingent groups, respectively). Although no significant interaction effect was observed, planned

comparisons between contingent and noncontingent groups for each coping style revealed a significant training effect in the flexible coping group.

relevant neural responses and interactions associated with behavioral training. Corroborating previous findings in our lab, the current findings emphasize the potential value of strategic cognitive-behavioral training programs for shaping adaptive neural responses in the midst of uncertainty (Bardi et al., 2012, 2013). Among the neural responses investigated, the roles of differential expression of neuroplasticity in the hippocampus, as well as complex involvement of the lateral habenula, emerged as potentially influential responses to uncertain conditions in the current data set.

Focusing on the animals' behavior in response to the prediction error in the probe trial, the effect of EBR training (as opposed to coping strategies) had the most significant impact on the animals' responses. The C-T animals spent more time in proximity to the previously baited well and, additionally, exhibited more rearing responses in the arena. Alternatively, the NC-T animals visited more wells in the arena and exhibited an increased number of interrupted grooming bouts. The only behavioral variable impacted by coping style was exploration in the arena. In this case, coping style interacted with contingency-training so that, although training didn't differentiate the active and passive copers, it was associated with decreased exploration in the flexible copers. Thus, the behavioral data suggest that the C-T animals utilized a more strategic search strategy - targeting the most recently baited well and exhibiting more rearing responses, providing animals with an opportunity to scan the environment for potentially relevant cues from a different vantage point. Alternatively, the NC-T animals exhibited a more general, less specific search by visiting more of the wells, regardless of their reinforcement history. The effect of training on grooming suggests that NC-trained animals had a heightened stress response relative to the C-T animals (Bardi et al., 2011). Namely, increased interruptions of grooming bouts in the NC-T animals suggest that these rats possessed less emotional regulation than the contingent-trained animals. This observation is reinforced by the endocrine data collected several days prior to the probe trial, indicating that the trained animals had higher DHEA/CORT ratios, an index of emotional resilience (Karishma and Herbert, 2002; Morgan et al., 2004; Yehuda et al., 2006; Feder et al., 2009; Bardi et al., 2012). Thus, these findings indicate that, in addition to the strategic responses exhibited in the presence of uncertain contextual contingencies, the contingency trained animals demonstrated increased emotional regulation. It is important to point out that the DLM acquisition trial, although challenging in that it was the first trial with only one food reward available in the food wells, did not appear to be any more stressful than the early stages of the EBR training. Thus, it would be interesting to explore endocrine responses in more challenging situations to understand more about the effects of EBR training on allostasis and emotional regulation in various stressful contexts. A limitation of the current study was that fecal samples, which need to be collected $12 \mathrm{~h}$ following the event of interest to detect the endocrine peak, could not be collected following the probe trial due to the need to process the brain tissue shortly after the trial for fos-immunoreactivity.

The pattern of neural responsiveness in the varying coping and training groups, not surprisingly, is complex. Nonetheless, certain patterns emerged in the current data set. As described earlier, the lateral habenula appears to play an important role in an animal's responses to uncertainty (Li et al., 2011); however, results of the current study suggest that its impact on uncertainty in the DLM needs further exploration. A significant inverse correlation between DHEA (during the DLM acquisition trial) and fos-immunoreactivity in the habenula following the probe test indicates a lower activation in this area in the animals exhibiting the most resilient endocrinological profile. This finding corroborates previous research characterizing the lateral habenula as playing a role in behavioral suppression in the context of perceived threats (Geisler and Trimble, 2008), with increased activation leading to diminished behavioral flexibility (Geisler and Trimble, 2008; Bianco and Wilson, 2009). Additionally, although not statistically significant, flexible animals had a higher ratio of neurons to glia than the consistent passive and active copers and this variable was relevant in the MDS analysis. If corroborated in future studies, this finding suggests that, in the flexible animals, fewer glial cells relative to neurons may be present in the laberal habenula. Increased glial cells per neuron may contribute to heightened reactivity in this area, leading to disrupted emotional regulation and top-down processing necessary for strategic responses. When the neuroanatomy of the laberal habenula is considered, its relevance in the symptoms of depression becomes apparent. Processes extending from the lateral habenula to the raphe nuclei influence the serotonergic system that is so frequently implicated in depression symptoms and treatment; further, the habenular efferents exert tonic inhibition on dopaminergic neurons in the ventral tegmental area, potentially 


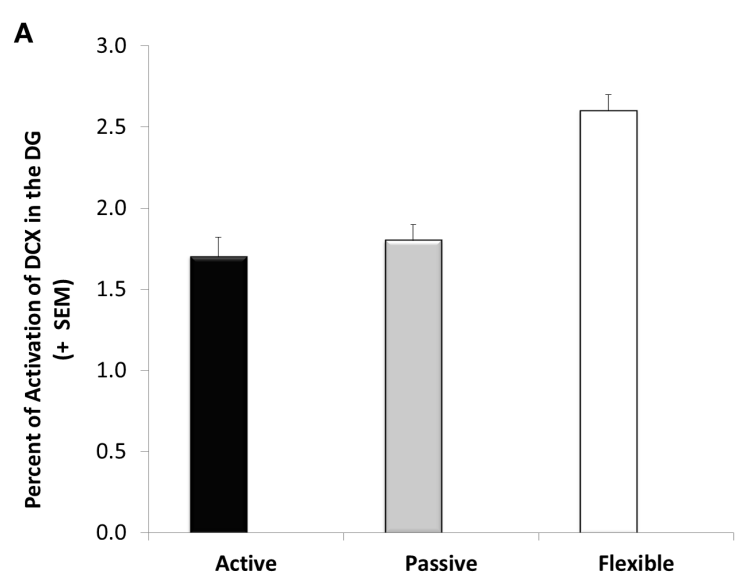

FIGURE 5 | Neuroplasticity markers. Doublecortin-immunoreactive tissue was higher in the flexible coping animals than their active and passive counterparts (A) whereas a significant interaction was observed in the
B

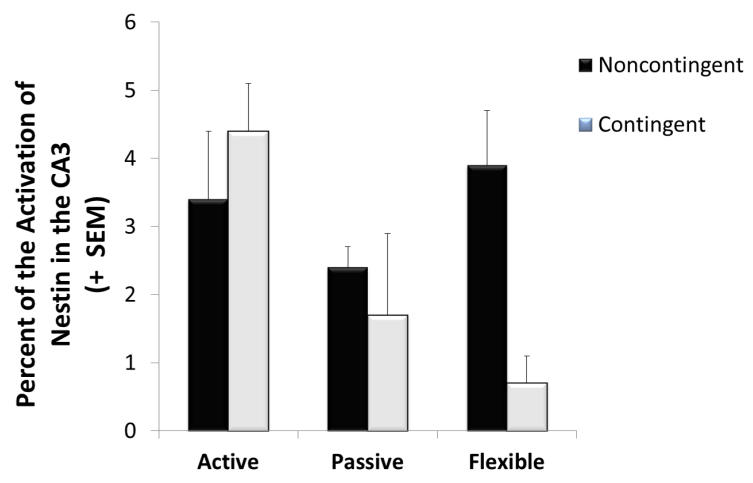

Nestin-immunoreactive tissue (B); specifically, lower levels were observed in the flexible contingent trained animals than in the flexible noncontingent animals, an effect not observed in the active and passive coping groups.
Table 2 | Correlations among neural activation and hormonal metabolites levels after the dry land maze (DLM) acquisition trial.

\begin{tabular}{|c|c|c|c|}
\hline $\begin{array}{l}\text { Hormone levels after } \\
\text { DLM acquisition trial }\end{array}$ & CORT & DHEA & DHEA/CORT \\
\hline DCX-ir (DG) & $\begin{array}{l}-0.193 \\
\text { ns }\end{array}$ & $\begin{array}{c}0.166 \\
\text { ns }\end{array}$ & $\begin{array}{c}0.358^{*} \\
(p=0.037)\end{array}$ \\
\hline Nestin (CA2) & $\begin{array}{c}0.341^{*} \\
(p=0.048)\end{array}$ & $\begin{array}{c}0.052 \\
\mathrm{~ns}\end{array}$ & $\begin{array}{l}-0.203 \\
\text { ns }\end{array}$ \\
\hline Fos-ir (habenula) & $\begin{array}{l}-0.206 \\
n s\end{array}$ & $\begin{array}{c}-0.373^{*} \\
(p=0.030)\end{array}$ & $\begin{array}{c}-0.292^{\wedge} \\
(p=0.094)\end{array}$ \\
\hline BDNF (habenula) & $\begin{array}{c}-0.326^{\wedge} \\
(p=0.060)\end{array}$ & $\begin{array}{c}-0.122 \\
n s\end{array}$ & $\begin{array}{l}-0.011 \\
\text { ns }\end{array}$ \\
\hline \multicolumn{4}{|c|}{${ }^{*} p$-value $<0.05 ; \wedge p$-value $<0.10$} \\
\hline \multicolumn{4}{|c|}{$\begin{array}{l}\text { Table } 3 \text { | Corticosterone, DHEA, and DHEA/Corticosterone ratios by } \\
\text { coping and training. }\end{array}$} \\
\hline $\begin{array}{l}\text { Hormone levels after } \\
\text { DLM acquisition trial }\end{array}$ & $\begin{array}{l}\text { CORT (1.1 g/ } \\
0.1 \mathrm{~g} \text { feces) }\end{array}$ & $\begin{array}{l}\text { DHEA (pg/ } \\
0.1 \mathrm{~g} \text { feces) }\end{array}$ & DHEA/CORT \\
\hline Flexible & 834 & 1835 & 4.008 \\
\hline Active & 886 & 1192 & 1.481 \\
\hline Passive & 872 & 1305 & 2.235 \\
\hline Contingent & 606 & 1522 & 3.695 \\
\hline Non-contingent & 1125 & 1320 & 1.286 \\
\hline
\end{tabular}

affecting pleasure and reward experiences that characterize the symptoms of depression (Lecourtier et al., 2008; Bianco and Wilson, 2009). Functionally, the lateral habenula conveys information about prediction errors to the mesolimbic dopaminergic area of the brain, leading to a significant impact on learning responses. Suppressing lateral habenula activity with a GABA antagonist causes increases in DA release in the nucleus accumbens and striatum in a similar time course and magnitude to those

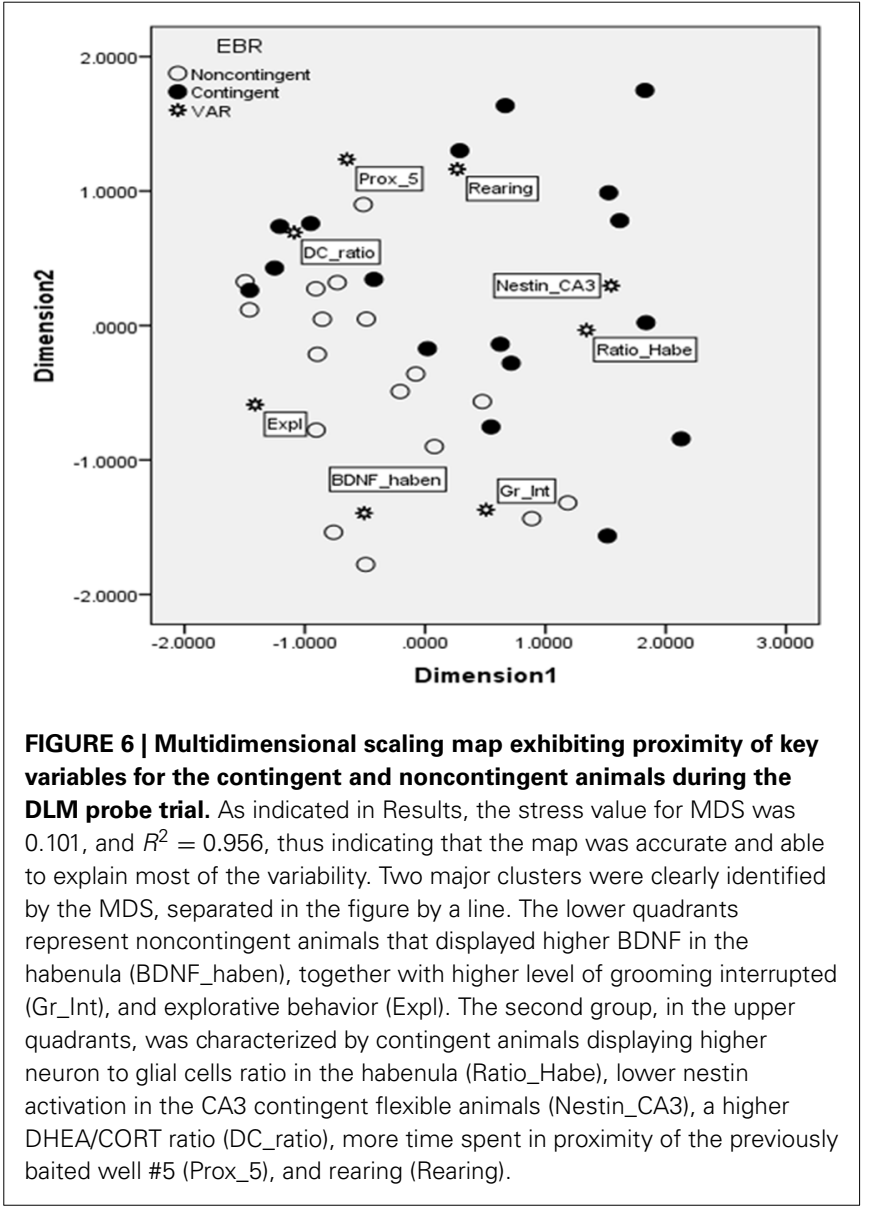

observed during reward-seeking behavior (Bianco and Wilson, 2009). Considering its interactions with both the serotonergic and dopaminergic systems, as well as its input from both the limbic system and the medial prefrontal cortex, the lateral habenula can be envisioned as a neuroanatomical hub of the constellation of 
symptoms consistent with depression (e.g., disrupted reward processing, goal-seeking, and strategic action-outcome processing) (Geisler and Trimble, 2008; Matsumoto and Hidosaka, 2009; Li et al., 2011; Henn, 2012).

Altered patterns of neuroplasticity have also been considered to be influential in the emergence of depressive symptoms. Increased BDNF (a neurotrophic factor), as well as increased rates of neurogenesis, have been associated with the effectiveness of antidepressants (Malberg et al., 2000; Santarelli et al., 2003; De Foubert et al., 2004). In the current study, two measures of neuroplasticity, including doublecortin- and nestinimmunoreactivity were influenced more by coping profiles than training. Namely, the flexible animals had higher levels of doublecortin-immunoreactivity (an index of cellular proliferation) in the dentate gyrus and the flexible contingent-trained animals exhibited decreased levels of nestin-immunoreactivity (an index of restructuring of mature cells in this case) in the hippocampal CA3 area, but not in the CA1 and CA2 areas (Hendrickson et al., 2011). These divergent findings of neuroplastic functions in the same group of animals confirm the complexity of neuroplasticity and the importance of monitoring several indices to obtain the complete picture of the brain's neuroplastic profile. It has been suggested that BDNF may be differentially involved with specific aspects of depression symptoms (Duman and Monteggia, 2006; Martinowich et al., 2007); accordingly, the coping profiles and behavioral training used in the current study provide an opportunity for systematic investigations of several measures of neuroplasticity.

Using both statistical findings in the current study and theoretical inferences based on past findings reported in the literature, a MDS model was built to further understand the relationships among the variables differentiating C-T and NC-T animals. Specifically, the strategies exhibited by the contingent animals, characterized by spending more time in proximity to the previously baited well and engaging in more frequent rearing responses, appear to be strategic responses in that they are associated with accurate memories of expected rewards (proximity to baited well) and the acquisition of increased information (increased rearing) to potentially resolve conflicts associated with uncertainty arising from the prediction error. From a neuroanatomical view, less restructuring of the mature CA3 neurons (determined by nestin-immunoreactive cells) and fewer glial cells servicing the lateral habenula neurons (suggested by the neuron/glia ratio measures), appear to be related to the strategic responses of the contingent-trained animals and, considering the training effect on the DHEA/CORT ratio, emotional regulation in this study. Thus, these results corroborate previous research suggesting that decreased habenular activity leads to enhanced goal seeking (Bianco and Wilson, 2009); however, the lack of a significant effect on fos-activation suggests that the role of the habenula in response to uncertainty in the DLM is complex. On the other hand, the noncontingent animals were associated with increased exploration across the entire arena and more interrupted grooming sequences. Although the exploration of additional wells may be adaptive in the sense that they once contained food rewards, the increased energy expenditure and disrupted grooming appeared as less targeted and efficient than the contingent-trained animals' strategies. However, additional research is necessary to further clarify behaviors that are consistent with strategic and adaptive responses in specific contexts. Finally, the close proximity of habenula BDNF reactivity with the noncontingent animals in the MDS model provides further support of the potential role of this structure, as well as the role of neuroplasticity, in emotional regulation and performance in cognitive tasks in uncertain conditions.

In sum, the current study confirms that the constellation of symptoms that have been associated with compromised emotional resilience and increased susceptibility to depression can be investigated in tasks involving emotional challenges such as uncertainty, ambiguity and prediction errors. Although cognitive in nature, the probe trial of the Dry Land maze also provides a view of various responses in the presence of uncertainty. In this case, the apparently mild level of stress associated with the absence of the expected reward allows for a more systematic evaluation of the interactions of the limbic and cognitive processes in the evaluation of changing contingencies. As reported above, these findings suggest that, although predisposed coping strategies are important and may be associated with a neural advantage (i.e., cellular proliferation) facilitating strategic decision-making, this advantage is tempered by behavioral training and an animal's relevant contingency history. Further research is necessary, however, to identify the specific neurobiological underpinnings of emotional resilience and how an organism's contingency history and predisposed coping strategies can contribute to enhanced adaptive functioning and diminished vulnerability to stress-related psychiatric diseases such as depression.

\section{AUTHOR CONTRIBUTIONS}

Kelly G. Lambert designed the study and supervised all aspects of data collection, as well as writing the manuscript. Massimo Bardi conducted the endocrinological assays and statistical analyses. Molly M. Hyer and Amanda A. Rzucidlo conducted the behavioral training and trials, histology and neural quantification. Timothy Bergeron and Timothy Landis also contributed to the neural quantification.

\section{ACKNOWLEDGMENTS}

This research was supported by the Chenery Research Professorship funds (to KGL) and Randolph-Macon College.

\section{REFERENCES}

Abramson, L. Y., Seligman, M. E. P., and Teasdale, J. D. (1978). Learned helplessness in humans: critique and reformation. J. Abnorm. Psychol. 87, 49-74. doi: 10.1037/0021-843X.87.1.49

Alexander, W. H., and Brown, J. W. (2011). Medial prefrontal cortex as an actionoutcome predictor. Nat. Neurosci. 14, 1338-1344. doi: 10.1038/nn.2921

Bardi, M., Franssen, C. L., Hampton, J. E., Shea, E. A., Fanean, A. P., and Lambert, K. G. (2011). Paternal experience and stress responses in the California mouse (Peromyscus californicus). Comp. Med. 60, 20-23.

Bardi, M., Rhone, A. P., Franssen, C. L., Hampton, J. E., Shea, E. A., Hyer, M. M., et al. (2012). Behavioral training and predisposed coping strategies interact to influence resilience inmale Long-Evans rats: implications for depression. Stress 15, 306-317.

Bardi, M., True, M., Franssen, C. L., Kaufman, C., Rzucidlo, A., and Lambert, K. G. (2013). Effort-based reward (EBR) training enhances neurobiological efficiency 
in a problem-solving task: insights for depression therapies. Brain Res. 1490, 101-110. doi: 10.1016/j.brainres.2012.10.027

Behrens, T. E. J., Woolrich, M. W., Walton, M. E., and Rushworth, M. F. S. (2007). Learning the value of information in an uncertain world. Nat. Neurosci. 10, 1214-1221. doi: 10.1038/nn1954

Bianco, I. H., and Wilson, S. W. (2009). The habenular nuclei: a conserved asymmetric relay station in the vertebrate brain. Philos. Trans. R. Soc. Lond. B Biol. Sci. 364, 1005-1020. doi: 10.1098/rstb.2008.0213

Bubic, A., Yves von Cramon, D., and Schubotz, R. I. (2010). Prediction, cognition and the brain. Front. Hum. Neurosci. 4:25. doi: 10.3389/fnhum.2010.00025

Bush, G., Luu, P., and Posner, M. I. (2000). Cognitive and emotional influences in anterior cingulate cortex. Trends Cogn. Sci. 4, 215-222. doi: 10.1016/S13646613(00)01483-2

Cavigelli, S. A., and McClintock, M. K. (2003). Fear of novelty in infant rats predicts adult corticosterone dynamics and an early death. Proc. Natl. Acad. Sci. U.S.A. 100, 16131-16136. doi: 10.1073/pnas.2535721100

Cavigelli, S. A., Stine, M. M., Kovacsics, C., Jefferson, A., Diep, M. N., and Barrett, C. E. (2007). Beahvioral inhibition and glucocorticoid dynamics in a rodent model. Physiol. Behav. 92, 897-905. doi: 10.1016/j.physbeh.2007.06.016

Colby, D. A., and Shifren, K. (2013). Optimism, mental health, and quality of life: a study among breast cancer patients. Psychol. Health Med. 18, 10-20. doi: 10.1080/13548506.2012.686619

Damasio, A. R., Grabowski, T. J., Bechara, A., Damasio, H., Ponto, L. L. B., Parvizi, J., et al. (2000). Subcortical and cortical brain activity during the feeling of selfgenerated emotions. Nat. Neurosci. 3, 1049-1056. doi: 10.1038/79871

De Foubert, G., Carney, S. L., Robinson, C. S., Destexhe, E. J., Tomlinson, R., Hicks, C. A., et al. (2004). Fluoxetine-induced change in rat brain expression of brain-derived neurotrophic factor varies depending on length of treatment. Neuroscience 128, 597-604. doi: 10.1016/j.neuroscience.2004.06.054

Dimidjian, S., Barrera, M., Martell, C., Murioz, R. F., and Lewinsohn, P. M. (2011). The origins an current status of behavioral activation treatments for depression. Annu. Rev. Clin. Psychol. 7, 1-38. doi: 10.1146/annurev-clinpsy-032210-104535

Donegan, J. J., Girotti, M., Weinberg, M. S., and Morilak, D. A., (2013). A novel role for brain interleukin-6: facilitation of cognitive flexibility in rat orbitofrontal cortex. J. Neurosci. 34, 953-962. doi: 10.1523/JNEUROSCI.3968-13.2014

Duman, R. S., and Monteggia, L. M. (2006). A neurotrophic model for stressrelated mood disorders. Biol. Psychiatry 59, 1116-1127. doi: 10.1016/j.biopsych. 2006.02.013

Egner, T. (2011). Surprise! A unifying model of dorsal anterior cingulate functions? Nat. Neurosci. 10, 1219-1220. doi: 10.1038/nn.2932

Endepols, H., Sommer, S., Backs, H., Wiedermann, D., Garf, R., and Huber, W. (2010). Effort-based decision making in the rat: a [18F] Fluorodeoxyglucose mocro position emission tomography study. J. Neurosci. 23, 1177-1187.

Feder, A., Nestler, E. J., and Charney, D. S. (2009). Psychobiology and molecular genetics of resilience. Nat. Rev. Neurosci. 10, 446-457. doi: 10.1038/nrn2649

Franssen, C. L., Bardi, M., Shea, E., Hampton, J. E., Franssen, R. A., Kinsley, C. H., et al. (2011). Fatherhood enhances learning and associated neural responsiveness. J. Neuroendocrinol. 23, 1177-1187. doi: 10.1111/j.1365-2826.2011. 02225.x

Gabriel, M., and Talk, A. C. (2001). "A tale of two paradigms: lessons learned from parallel studies of discriminative instrumental learning and classical eyeblink conditioning," in Model Systems and the Neurobiology of Associative Learning, eds J. A. Steinmetz, M. Gluck, and R. R. Soloman (Mahwah, NJ: Erlbaum), 149-185.

Geisler, S., and Trimble, M. (2008). The lateral habenula: no longer neglected. CNS Spectr. 13, 484-489.

Hawley, D. F., Bardi, M., Everette, A. M., Higgins, T. J., Tu, K. M., Kinsley, C. H., et al. (2010). Neurobiological constituents of active, passive, and variable coping strategies in rats: integration of regional brain neuropeptide $\mathrm{Y}$ levels and cardiovascular responses. Stress 13, 172-183. doi: 10.3109/10253890903144621

Hendrickson, M. L., Rao, A. J., Demerdash, O. N. A., and Kalil, R. E. (2011). Expression of nestin by neural cells in the adult rat and human brain. PLoS ONE 6:e18535. doi: 10.1371/journal.pone.0018535

Henn, F. R. A. (2012). Circuits, cells, and synapses: toward a new target for deep brain stimulation in depression. Neuropsychopharmacol. Rev. 37, 307-308. doi: 10.1038/npp.2011.193

Johnson, D. M., Liig, K. R., Behan, M., and Haberly, L. B. (2000). New features of connectivity in piriform cortex visualized by intracellular injection of pyramidal cells suggest that "primary" olfactory cortex functions like "association" cortex in other sensory systems. J. Neurosci. 20, 6974-6982.
Karishma, K. K., and Herbert, J. (2002). Dehydroepiandrosterone (DHEA) stimulates neurogenesis in the hippocampus of the rat, promotes survival of newly formed neurons and prevents corticosterone-induced suppression. Eur. J. Neurosci. 16, 445-453. doi: 10.1046/j.1460-9568.2002. 02099.x

Klanker, M., Feenstra, M., and Denys, D. (2013). Dopaminergic control of cognitive flexibility in humans and animals. Front. Neurosci. 7:201. doi: 10.3389/fnins.2013.00201

Koolhaas, J. M., Bartolomucci, A., Buwalda, B., deBoer, S. F., Flugge, G., Korte, S. M., et al. (2011). Stress revisted: a critical evaluation of the stress concept. Neurosci. Biobehav. Rev. 35, 1291-1301. doi: 10.1016/j.neubiorev.2011.02.003

Koolhaas, J. M., deBoer, S. F., Buwalda, B., and Vanreenen, K. (2007). Individual variation in coping with stress: a multidimensional approach of ultimate and proximate mechanisms. Brain Behav. Evol. 70, 218-226. doi: 10.1159/00010 5485

Koolhaas, J. M., Korte, S. M., deBoer, S. F., Van Der Vegt, B. J., Van Reenen, C. G., Hopster, H., et al. (1999). Coping styles in animals: current status in behavior and stress physiology. Neurosci. Biobehav. Rev. 23, 925-935. doi: 10.1016/S01497634(99)00026-3

Krishnan, V., and Nestler, E. J. (2013). The molecular neurobiology of depression. Nature 455, 894-902. doi: 10.1038/nature07455

Kruskal, J. B., and Wish, M. (1978). Multidimensional Scaling. Beverly Hills, CA: Sage Publications.

Lambert, K. G. (2006). Rising rates of depression in today's society: consideration for the roles of effort-based rewards and enhanced resilience in day-to-day functioning. Neurosci. Biobehav. Rev. 30, 319-324. doi: 10.1016/j.neubiorev.2005. 09.002

Lecourtier, L., Defrancesco, A., and Moghaddam, B. (2008). Differential tonic influence of lateral habenula on prefrontal cortex and nucleus accumbens dopamine release. Eur. J. Neurosci. 27, 1755-1762. doi: 10.1111/j.1460-9568.2008. 06130.x

Li, B., Piriz, J., Mirrione, M., Chung, C., Proulx, C. D., Schulz, D., et al. (2011). Synaptic potentiation onto habenula neurons in learned helplessness model of depression. Nature 470, 535-539. doi: 10.1038/nature09742

Liljeholm, M., Tricomi, E., O’Doherty, J. P., and Balleine, B. W. (2011). Neural correlates of instrumental contingency learning: differnetial effects of actionreward conjunction and disjunction. J. Neurosci. 16, 2474-2480.

Malberg, J. E., Eisch, A. J., Nestler, E. J., and Duman, R. S. (2000). Chronic antidepressant treatment increases neurogenesis in the adult rat hippocampus. J. Neurosci. 20, 9104-9110.

Manly, B. F. (1994). Multivariate Statistical Methods. London: Chapman \& Hall.

Martinowich, K., Manji, H., and Lu, G. (2007). New insights into BDNF function in depression and anxiety. Nat. Neurosci. 10, 1089-1093. doi: 10.1038/nn1971

Matsumoto, K., and Tanaka, K. (2004). The role of the medial prefrontal cortex in achieving goals. Curr. Opin. Neurobiol. 14, 178-185. doi: 10.1016/j.conb.2004.03.005

Matsumoto, M., and Hidosaka, O. (2009). Representation of negative motivational value in the primate lateral habenula. Nat. Neurosci. 12, 77-84. doi: $10.1038 / \mathrm{nn} .2233$

McGuire, J. L., Larke, L. E., Sallee, F. R., Hermann, J. P., and Sah, R. (2011). Differential regulation of neuropeptide $\mathrm{Y}$ in the amygdala and prefrontal cortex during recovery from chronic variable stress. Front. Behav. Neurosci. 5:54. doi: 10.3389/fnbeh.2011.00054

McRae, K., Misra, S., Prasad, A. K., Pereira, S. C., and Gross, J. J. (2012). Bottomup and top-down emotion generation: implications for emotion regulation. Soc. Cogn. Affect. Neurosci. 7, 253-262. doi: 10.1093/scan/nsq103

Moore, J. W., Lagnado, D., Deal, D. C., and Haggard, P. (2009). Feelings of control: contingency determines experience of action. Cognition 110, 279-283. doi: 10.1016/j.cognition.2008.11.006

Morgan, C. A., Rasmusson, A., Pietrzak, R. H., Coric, V., and Southwick, S. M. (2009). Relationships among plasma dehydroepiandrosterone and degydroepiandrosterone sulfate, cortisol, symptoms of dissociation, and objective performance in humans exposed to underwater navigation stress. Biol. Psychiatry 66, 334-340. doi: 10.1016/j.biopsych.2009.04.004

Morgan, C. A., Southwick, S., Hazlett, G., Rasmusson, A., Hoty, G., Zimolo, Z., et al. (2004). Relationships among plasma dehydroepiandrosterone sulfate and cortisol levels, symptoms of dissociation, and objective performance in humans exposed to acute stress. Arch. Gen. Psychiatry 61, 819-825. doi: 10.1001/archpsyc.61.8.819 
Overmier, J. B., and Seligman, M. E. P. (1967). Effects of inescapable shock upon subsequent escape and avoidance responding. J. Comp. Physiol. Psychol. 63, 28-33. doi: 10.1037/h0024166

Paxinos, G., and Watson, C. (2007). The Rat Brain in Stereotaxic Coordinates, 6th $E d n$. New York, NY: Academic Press.

Pothuizen, H. H., Aggleton, J. P., and Vann, S. D. (2008). Do rats with retrosplenial cortex lesions lack direction? Eur. J. Neurosci. 28, 2486-2498. doi: 10.1111/j.1460-9568.2008.06550.x

Ragozzino, M. E., and Rozman, S. (2007). The effect of rat anterior cingulate inactivation on cognitive flexibility. Behav. Neurosci. 121, 698-706. doi: 10.1037/0735-7044.121.4.698

Rebola, J., Castelhano, J., Ferreira, C., and Castelo-Branco, M. (2012). Functional parcellation of the operculo-insular cortex in perceptual decision-making: an fMRI study. Neuropsychologia 50, 3693-3701. doi: 10.1016/j.neuropsychologia. 2012.06.020

Rivalan, M., Valton, V., Series, P., Marchland, A. R., and Dellu-Hagedom, F. (2013). Elucidating poor decision-making in a rat gambling task. PLoS ONE 8:e82052. doi: 10.1371/journal.pone.0082052

Robinson, O. J., Overstreet, C., Charney, D. R., Vytal, K., and Grillon, C. (2013). Stress increases aversive prediction error signal in the ventral striatum. Proc. Natl. Acad. Sci. U.S.A. 110, 129-133. doi: 10.1073/pnas.1213923110

Rushworth, M. F. S., and Behrens, T. E. J. (2008). Choice, uncertainty and value in prefrontal and cingulate cortex. Nat. Neurosci. 11, 389-397. doi: $10.1038 / \mathrm{nn} 2066$

Santarelli, L., Saxe, M., Gross, C., Surget, A., Battaglia, F., Dulawa, S., et al. (2003). Requirement of hippocampal neurogenesis for the behavioral effects of antidepressants. Science 301, 805-809. doi: 10.1126/science.1083328

Savitz, J. B., Nugent, A. C., Bogers, W., Roioser, J. P., Bain, E. E., Neumeister, A., et al. (2011). Habenula volume in bipolar disorder and major depressive disorder: a high resolution magnetic resonance imaging study. Biol. Psychiatry 69, 336-343. doi: 10.1016/j.biopsych.2010.09.027

Seligman, M. E. P., and Maier, S. F. (1967). Failure to escape traumatic shock. J. Exp. Psychol. 74, 1-9. doi: 10.1037/h0024514

Selye, H. (1936). A syndrome produced by diverse noxious agents. Nature 138, 32. doi: $10.1038 / 138032 \mathrm{a} 0$

Southwick, S. M., and Charney, D. S. (2012). The science of resilience: implications for the preventionand treatment of depression. Science 338, 79-82. doi: $10.1126 /$ science. 1222942
Steinberg, E. E., Keiflin, R., Boivin, J. R., Witten, I. B., Keisseroth, K., and Janak, P. H. (2013). A causal link between prediction errors, dopamine neurons and learning. Nat. Neurosci. 16, 966-973. doi: 10.1038/nn.3413

Vann, S. D., and Aggleton, J. P. (2004). Testing the importance of the retrosplenial guidance system: effects of different sized retrosplenial cortex lesions on heading direction and spatial working memory. Behav. Brain Res. 155, 97-108. doi: 10.1016/j.bbr.2004.04.005

Vann, S. D., Aggleton, J. P., and Maguire, E. A. (2009). What does the retrosplenial cortex do? Nat. Rev. Neurosci. 10, 792-802. doi: 10.1038/nrn2733

Vann, S. D., Wilton, L. A., Muir, J. L., and Aggleton, J. P. (2003). Testing the importance of the caudal retrosplenial cortex for spatial memory in rats. Behav. Brain Res. 140, 107-118. doi: 10.1016/S0166-4328(02)00274-7

Wechsler, B. (1995). Coping and coping strategies: a behavioral view. Appl. Animal Behav. Sci. 43, 123-124. doi: 10.1016/0168-1591(95)00557-9

Wu, G., Feder, A., Cohen, H., Kim, J. J., Caleron, S., Charney, D. S., et al. (2013). Understanding resilience. Front. Behav. Neurosci. 7:10. doi: 10.3389/fnbeh.2013.00010

Yehuda, R., Braind, S. R., Colier, J. A., and Yang, R. K. (2006). Clinical correlates of DHEA associated with post-traumatic stress disorder. Acta Psychiatr. Scand. 114, 187-193. doi: 10.1111/j.1600-0447.2006.00801.x

Conflict of Interest Statement: The authors declare that the research was conducted in the absence of any commercial or financial relationships that could be construed as a potential conflict of interest.

Received: 22 November 2013; accepted: 24 March 2014; published online: 28 April 2014.

Citation: Lambert KG, Hyer MM, Rzucidlo AA, Bergeron T, Landis T and Bardi $M$ (2014) Contingency-based emotional resilience: effort-based reward training and flexible coping lead to adaptive responses to uncertainty in male rats. Front. Behav. Neurosci. 8:124. doi: 10.3389/fnbeh.2014.00124

This article was submitted to the journal Frontiers in Behavioral Neuroscience.

Copyright (c) 2014 Lambert, Hyer, Rzucidlo, Bergeron, Landis and Bardi. This is an open-access article distributed under the terms of the Creative Commons Attribution License (CC BY). The use, distribution or reproduction in other forums is permitted, provided the original author(s) or licensor are credited and that the original publication in this journal is cited, in accordance with accepted academic practice. No use, distribution or reproduction is permitted which does not comply with these terms. 